\title{
Electronic, dielectric, and optical properties of two-dimensional and bulk ice: A multiscale simulation study
}

\author{
S. Ghasemi, ${ }^{1}$ M. Alihosseini, ${ }^{2}$ F. Peymanirad, ${ }^{3}$ H. Jalali, ${ }^{2}$ S. A. Ketabi, ${ }^{1}$ F. Khoeini, ${ }^{2}$ and M. Neek-Amal $\oplus^{3,4,}{ }^{*}$ \\ ${ }^{1}$ School of Physics, Damghan University, Damghan, 3671641167 Semnan, Iran \\ ${ }^{2}$ Department of Physics, University of Zanjan, 45195-313 Zanjan, Iran \\ ${ }^{3}$ Department of Physics, Shahid Rajaee Teacher Training University, 16875-163 Lavizan, Tehran, Iran \\ ${ }^{4}$ Department of Physics, Universiteit Antwerpen, Groenenborgerlaan 171, B-2020 Antwerpen, Belgium
}

(Received 8 January 2020; revised manuscript received 16 March 2020; accepted 15 April 2020;

published 6 May 2020)

\begin{abstract}
The intercalated water into nanopores exhibits anomalous properties such as an ultralow dielectric constant. Multiscale modeling and simulations are used to investigate the dielectric properties of various crystalline two-dimensional ices and bulk ices. Although the structural properties of two-dimensional (2D) ices have been extensively studied, much less is known about their electronic and optical properties. First, by using density functional theory and density functional perturbation theory (DFPT), we calculate the key electronic, optical, and dielectric properties of 2D ices. Performing DFPT calculations, both the ionic and electronic contributions of the dielectric constant are computed. The in-plane electronic dielectric constant is found to be larger than the out-of-plane dielectric constant for all the studied 2D ices. The in-plane dielectric constant of the electronic response $\left(\varepsilon_{e l}\right)$ is found to be isotropic for all the studied ices. Second, we determined the dipolar dielectric constant of $2 \mathrm{D}$ ices using molecular dynamics simulations at finite temperature. The total out-of-plane dielectric constant is found to be larger than 2 for all the studied 2D ices. Within the framework of the random-phase approximation, the absorption energy ranges for $2 \mathrm{D}$ ices are found to be in the ultraviolet spectra. For comparison purposes, we also elucidate the electronic, dielectric, and optical properties of four crystalline ices (ice VIII, ice $\mathrm{XI}$, ice Ic, and ice Ih) and bulk water.
\end{abstract}

DOI: 10.1103/PhysRevB.101.184202

\section{INTRODUCTION}

The phase behavior of two-dimensional (2D) ice is the subject of recent experimental and theoretical interest, and is still controversial [1-8]. Recently the report of the observation of monolayer, bilayer, and trilayer ice using transmission electron microscopy [1] was challenged [9]; however, several theoretical studies based on both classical force fields and ab initio simulations revealed the exciting possibility of exploring 2D-ice structures at specific conditions [2,3,10,11]. In particular, both classical force fields and ab initio simulations predict that water molecules form the ordered flat-square lattice structure while they are trapped in a slit of a few angstroms in size $[2,10]$. The confinement width needed for the formation of stable monolayer ice is approximately $h \simeq$ 5-7 ̊ $[1,10,11]$.

In contrast, the structural properties of $2 \mathrm{D}$ ice (and bulk ice) and the electronic, dielectric, and optical properties of 2D ice and nanoconfined water are not well understood. Therefore, a solid theoretical background for the effects of size reduction on the dielectric properties of ice and confined water is highly demanded. This can be helpful to understand the measured anomalous dielectric properties of confined water [12]; recently, by using electrostatic force detection in atomic force microscopy, unexpected variation in the out-of-plane

*Corresponding author: mehdi.neekamal@gmail.com dielectric constant of confined water between graphene and hexagonal boron nitride ( $h$-BN) has been observed [12]. The presence of an interfacial water layer (having ice phase) with vanishingly small polarization is the reason for such small out-of-plane dielectric constant $(\simeq 2)$ for channels with a size of $h<15 \AA$. Indeed the dielectric constant of nanoconfined water was found to be about 2, which is above the highfrequency dielectric constant of water, i.e., 1.8.

In the past few decades, molecular dynamics simulations (MDSs) and Monte Carlo simulations (MCSs) have been used to calculate the dipolar dielectric constant of water and confined water. For instance the variation of the dipolar dielectric constant with temperature and pressure for the ices Ih, III, V, VI, and VII was studied by Aragones et al. [13]. In addition to MDS and MCS methods, mean field theory (such as Kirkwood's theory) was also used and yielded valuable insights into the H-bonding effects on the water dielectric constant [14]. Notwithstanding the existing MD-based theory studies in the past few years, the questions about the ionic and electronic contributions of the dielectric constant of 2D ice are still unanswered. Most of the previous studies reported the dipolar dielectric constant of water confined at the nanoscale channels [15-17]. Thus, one naturally expects to quantify the dipolar, electronic, and ionic dielectric constants of crystalline 2D ice and the corresponding frequency dependence. This will provide a solid theoretical support for the recent experiment [12].

Here, we conduct a systematic study for determining the electronic, dielectric, and optical properties of $2 \mathrm{D}$ ice using 

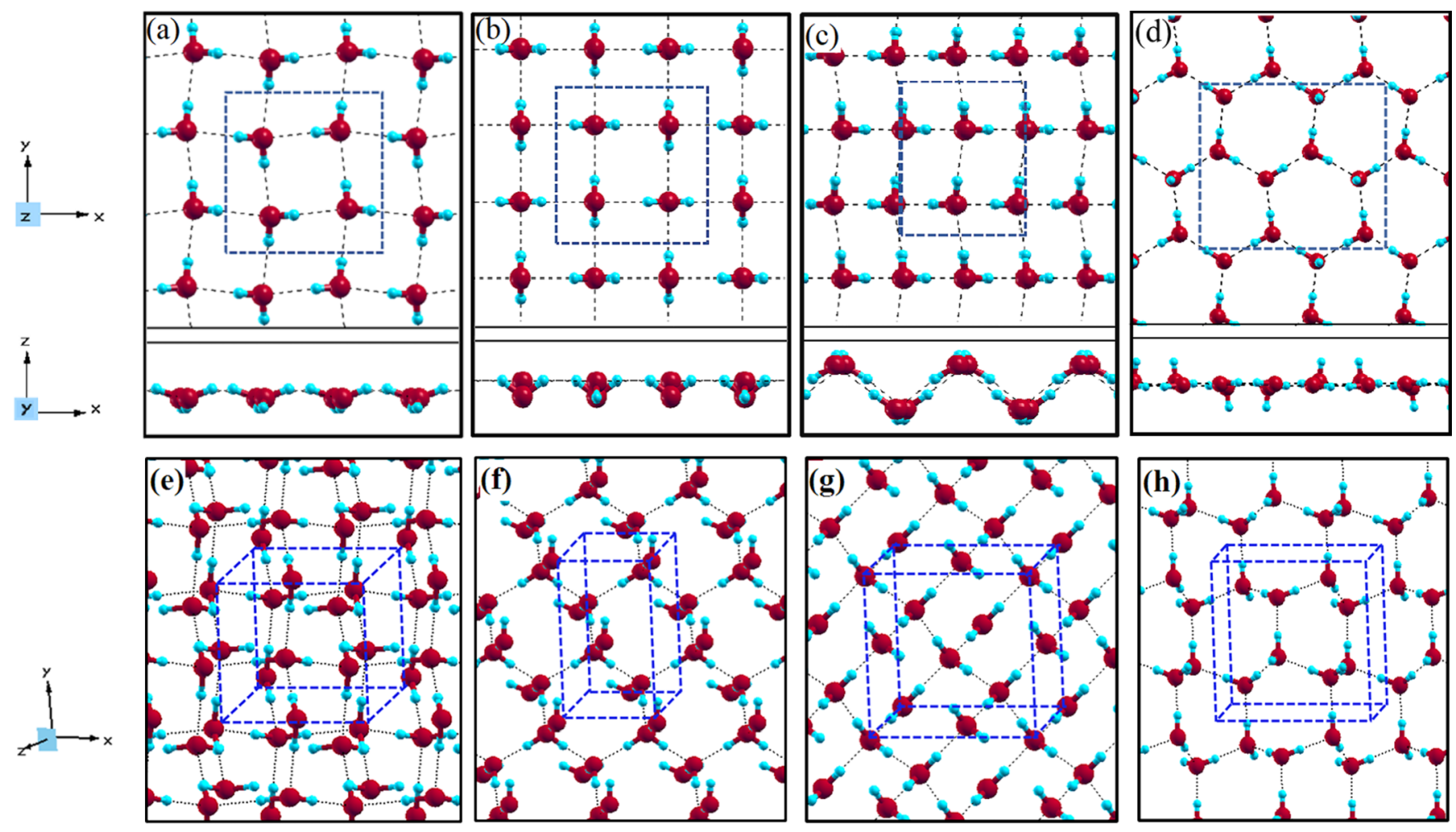

FIG. 1. The lattice structure of two-dimensional and bulk ices. The top and side views of (a) flat-square (f-SQ), (b) buckled-square (b-SQ), (c) buckled-rhombic (b-RH), and (d) hexagonal (HEX) 2D-ice structures. The blue squares show the corresponding supercells. (e) Ice VIII, (f) ice XI, (g) ice Ic, and (h) ice Ih. The blue cubes show the corresponding unit cells.

a multiscale approach including first-principles and molecular dynamics simulations. Both the out-of-plane and inplane components of ionic, electronic, and dipolar dielectric constants of the stable structure of 2D ices including the flat-square, buckled-square, buckled-rhombic, and hexagonal structures are investigated [2]. We also report results for the electronic, dielectric, and optical properties of the crystalline bulk ice and bulk water. Our work provides benchmark theoretical data for the electronic, dielectric, and optical properties of crystalline $2 \mathrm{D}$ and bulk ices.

\section{TWO-DIMENSIONAL ICE}

The 2D-ice structures considered in this paper are the flat-square (f-SQ), buckled-square (b-SQ), buckled-rhombic (b-RH), and hexagonal (HEX) structures. Their optimized structures were reported in Ref. [2]. The f-SQ and b-RH (HEX) structures have square (rectangular) unit cells containing 4 (8) water molecules [see Figs. 1(a)-1(d)]. In order to eliminate the interaction between periodic images in $a b$ initio calculations, we set a large vacuum with height $c$ for calculating each 2D ice (see Sec. IV). The lattice parameters of the simulation supercells $(a, b, c)$ are listed in Table I. The input lattice structures were extracted from the optimized structures of Ref. [2]. Using the van der Waals diameter of the oxygen atom, one can approximate the effective thickness $(t)$ of a given 2D-ice lattice: considering the flat structure of f-SQ, $t_{\mathrm{f} \text {-SQ }}=t_{0}=2 \times R_{v}$, where $R_{v}$ is the van der Waals radius of the oxygen atom. For b-SQ and HEX structures, there is $1 \AA$ difference between the top row and bottom row of $\mathrm{O}$ atoms [see side view of b-SQ and HEX structures in Figs. 1(b) and 1 (d)]; thus $t_{\mathrm{b}-\mathrm{SQ}, \mathrm{HEX}}=1+t_{0}$. For the b-RH, there is a large ( 4 $\AA$ ) distance between the top row and bottom row of $\mathrm{O}$ atoms, i.e., $t_{\mathrm{b}-\mathrm{RH}}=4+t_{0}$. All the structure parameters of the studied systems and the important findings of this paper are tabulated in Table I.

\section{CRYSTALLINE BULK ICE}

In order to compare electronic, dielectric, and optical properties of above-mentioned 2D ices with the high-pressure phase of ice, we also calculated the dielectric constant of four crystalline ices, i.e., cubic (ice VIII and ice Ic) and hexagonal (ice XI and ice Ih) bulk ices. The ice-XI, ice-Ic, and ice-Ih structures have 8 water molecules per orthorhombic unit cell [see Figs. 1(e)-1(h)]. The lattice parameters of the crystalline ices are listed in Table I. The ice Ih has the hexagonal crystalline form of ordinary ice, which is stable at temperature $273 \mathrm{~K}$ (down to a few kelvins) and pressures up to $200 \mathrm{MPa}$. The ice Ic is one of the metastable cubic crystalline form of ice, which is stable at temperatures between 130 and $220 \mathrm{~K}$. The ice VIII with 8 water molecules per unit cell has a tetragonal crystalline form and is stable under high pressures of about $3 \mathrm{GPa}$ below $278 \mathrm{~K}$. The ice XI is a hydrogen-ordered form of ice Ih containing 8 molecules per unit cell and is stable at temperature $5 \mathrm{~K}$ and pressures of about $100 \mathrm{MPa}$. We also calculated the dielectric constant of bulk water using 17 water molecules inside a 
TABLE I. Simulation parameters used for the calculation of dielectric constant of 2D ices and bulk ices: ionic dielectric constant ( $\varepsilon_{\text {ion }}$ ), the electronic dielectric constant $\left(\varepsilon_{\mathrm{el}}\right)$, the dipolar dielectric constant $\left(\varepsilon_{\mathrm{dip}}\right)$, total dielectric constant $\left(\varepsilon_{\text {tot }}\right)[41]$, the energy gap [ $\left.\Delta(\mathrm{eV})\right]$, and the effective thickness $(t)$. For comparison purposes we show the corresponding data for bulk water, TMDs, and $h$-BN. The used temperatures in the molecular dynamics simulations are given too.

\begin{tabular}{|c|c|c|c|c|c|c|c|c|c|c|c|c|c|c|c|c|c|c|c|}
\hline Structure & $a$ & $b$ & $c$ & $t$ & $\Delta(\mathrm{eV})$ & $x x$ & $y y$ & $z z$ & $x x$ & $y y$ & $z z$ & $x x$ & $y y$ & $z z$ & $x x$ & $y y$ & $z z$ & $T(\mathrm{~K})$ & Reference \\
\hline \multicolumn{20}{|l|}{ 2D ice: } \\
\hline f-SQ & 5.84 & 5.84 & 15 & $\left(t_{0}=\right) 3$ & 5.49 & 2.0 & 2.0 & 1.7 & 1.0 & 1.0 & 2.0 & 1.55 & 1.54 & 2.41 & 3.55 & 3.54 & 5.11 & 80 & Present work \\
\hline b-SQ & 5.78 & 5.78 & 20 & 4 & 5.55 & 1.8 & 1.8 & 1.49 & 3.85 & 3.9 & 0.01 & & & & & & & Ref. [28] & Present work \\
\hline \multicolumn{20}{|c|}{ 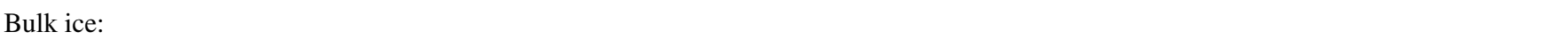 } \\
\hline Ice VIII & 4.73 & 4.73 & 6.85 & & 5.37 & 2.36 & 2.36 & 2.34 & 3.96 & 3.96 & 0.26 & 8.71 & 8.54 & 1.03 & 14.03 & 13.86 & 2.63 & 80 & Present work \\
\hline Ice XI & 4.45 & 7.7 & 7.27 & & 5.06 & 1.83 & 1.82 & 1.82 & 1.81 & 0.83 & 0.93 & 2.06 & 1.31 & 1.86 & 4.7 & 2.96 & 3.61 & 80 & Present work \\
\hline Ice Ic & 6.48 & 6.48 & 6.48 & & 4.99 & 1.77 & 1.77 & 1.76 & 1.21 & 1.21 & 0.16 & 2.1 & 2.1 & 1.01 & 4.08 & 4.08 & 1.93 & 80 & Present work \\
\hline Ice Ih & 7.81 & 7.38 & 4.52 & & 5.05 & 1.80 & 1.80 & 1.80 & 1.14 & 1.0 & 0.96 & 1.94 & 1.92 & 1.5 & 3.88 & 3.72 & 3.26 & 200 & Present work \\
\hline \multicolumn{20}{|l|}{ TMDs: } \\
\hline $\mathrm{MoS}_{2}$ & 3.21 & 3.21 & 32.1 & 6.12 & $<2$ & 15.1 & 15.1 & 6.4 & 0.2 & 0.2 & 0.0 & & & & & & & & Ref. [18] \\
\hline $\mathrm{WS}_{2}$ & 3.21 & 3.21 & 32.1 & 6.14 & $<2$ & 13.6 & 13.6 & 6.3 & 0.1 & 0.1 & 0.0 & & & & & & & & Ref. [18] \\
\hline $\mathrm{WSe}_{2}$ & 3.34 & 3.34 & 33.4 & 6.52 & $<2$ & 15.1 & 15.1 & 7.5 & 0.2 & 0.2 & 0.0 & & & & & & & & Ref. [18] \\
\hline
\end{tabular}

cubic unit cell with size $7.93 \times 7.93 \times 7.93 \AA^{3}$. We have used 20 different relaxed MDS configurations as inputs for the density functional theory calculations of bulk water. For the bulk water and bulk crystalline ices, we applied periodic boundary conditions in all directions, though for the 2D ices, a vacuum space must be set (see Sec. VA). In order to obtain more insights and for comparison purposes, we put in order the dielectric constant of some common 2D materials such as monolayer $h$-BN and monolayers of three transition metal dichalcogenides (TMDs) [18], i.e., $\mathrm{MoS}_{2}, \mathrm{WS}_{2}$, and $\mathrm{WSe}_{2}$.

\section{DIELECTRIC CONSTANT}

For the polar systems, the total dielectric constant tensor includes three main contributions, i.e., electronic, ionic, and dipolar:

$$
\varepsilon_{\text {total }}^{\mu \nu}=\varepsilon_{\mathrm{el}}^{\mu \nu}+\varepsilon_{\text {ion }}^{\mu \nu}+\varepsilon_{\mathrm{dip}}^{\mu \nu} .
$$

The indexes $\mu$ and $v$ run over the three spatial directions. At zero kelvins the dipolar term vanishes. By increasing the temperature of the 3D material, the dipolar term becomes important and should be taken into account. Note that different bulk ices are stable at different temperatures [19]. In Table I, the relevant temperatures are listed.

\section{THE METHODS}

In this study, density functional theory (DFT) has been implemented for electronic band structure calculations. We have used density functional perturbation theory (DFPT) to obtain the electronic and ionic dielectric constants at zero kelvins. Moreover, molecular dynamics simulations (MDSs) have been used to find the dipolar dielectric constant at a finite temperature for 2D (bulk) ices. In order to calculate the optical dielectric function, the random-phase approximation (RPA) [20] based on DFT ground-state calculations has been conducted. In the following sections, we briefly explain the different used methods.

\section{A. Density functional theory: Electronic band structure}

We have calculated the electronic band structure of 2D ices using DFT as implemented in the Quantum-ESPRESSO (QE) package [21]. We have used ultrasoft pseudopotentials to treat the interaction between the ion cores and valence electrons and applied the generalized gradient approximation for the exchange-correlation interactions. We also have studied the effect of nonlocal correlations using the van der Waals density functional of Dion et al. [22]. Accuracy of forces on each atom has been considered about $0.1 \mathrm{mRy} / \mathrm{bohr}$ for the variablecell optimization, relaxing the cell parameters and atomic positions. In order to accurately calculate the structural and electronic properties, kinetic energy cutoffs of 150 Ry and $1500 \mathrm{Ry}$ were found to be sufficient for the wave functions and charge densities, respectively, where the $k$-point grid for $2 \mathrm{D}$ (bulk) ices was set to $6 \times 6 \times 1(6 \times 6 \times 6)$. The $k$-point grid for $2 \mathrm{D}$ (bulk) ices was chosen as $24 \times 24 \times 1$ $(24 \times 24 \times 24)$ for non-self-consistent calculation in a partial density of states (PDOS) analysis. A smearing parameter of 0.01 Ry has been used for the PDOS analysis. In addition, 
the total energy convergence threshold was set to $10^{-12} \mathrm{eV}$. The Coulomb cutoff technique $[23,24]$ was used to reduce interactions between periodic images and the cost of the $a b$ initio calculations for $2 \mathrm{D}$-ice structures.

\section{B. Density functional perturbation theory: Electronic and ionic dielectric constant}

The dielectric properties of the 2D ices were determined using the DFPT approach. In DFPT, the dielectric constant tensor is defined as a linear response to the perturbative electric field $[25,26]$ and the ionic displacement is considered as a perturbation to the equilibrium system. The response of the electronic charge density to the perturbative electric field in the linear response regime is employed to determine the electronic contribution of the dielectric tensor, i.e., the highfrequency dielectric constant $\left(\varepsilon_{\mathrm{el}}\right)$. Subsequently, the static dielectric constant $\left(\varepsilon_{0}^{\mu \nu}\right)$ is the summation of the electronic and the ionic parts of the system to the applied electric field:

$$
\varepsilon_{0}^{\mu \nu}=\varepsilon_{\mathrm{el}}^{\mu \nu}+\varepsilon_{\mathrm{ion}}^{\mu \nu},
$$

where

$$
\varepsilon_{\mathrm{ion}}^{\mu \nu}=\frac{4 \pi}{\Omega} \sum_{m} \frac{S_{m}, \mu \nu}{\omega_{m}^{2}} .
$$

Here $S_{m}$ is the mode oscillator strength tensor, defined in terms of the Born effective charges $Z^{*}$, the atomic masses $M_{i}$, and the normalized eigenvectors $u_{i, m \mu}$ of the $i$ th ion along a given direction $\mu$ for a particular mode $m$. Also $\omega_{m}$ is the phonon mode frequency and $\Omega$ is the unit cell volume. Thus, in order to compute $\varepsilon_{0}$, the knowledge of all the phonon frequencies at the zone center of Brillouin zone is needed. The latter requires the solution of the dynamical matrix at the zone center.

\section{Molecular dynamics simulations: Dipolar dielectric constant}

By means of equilibrium molecular dynamics simulations employing the large-scale atomic/molecular massively parallel simulator LAMMPS [27], we computed the molecular (dipolar) dielectric constant of $2 \mathrm{D}$ ice at $80 \mathrm{~K}$. Our simulated systems contain 2D ices, confined between two walls, separated by a distance [2] $h=2 t$ (these walls are used to produce the confining potential). The number of water molecules is 400, 100, and 128 for f-SQ, R-SQ, and HEX, respectively [28]. The TIP4P model was employed to describe the water molecules [29]. The NVT ensemble (Nosé-Hoover thermostat) is used to keep the temperature at $80 \mathrm{~K}$ for $2 \mathrm{D}$ and bulk ices excluding ice Ih $(200 \mathrm{~K})$. We modified the TIP4P model to incorporate the lattice structure of the optimized structures by using DFT: we changed bond lengths and bond angles of water molecules relevant to the DFT outputs [2]. Also, the charges of the $\mathrm{O}$ and $\mathrm{H}$ atoms were modified such that a large dipole moment $(\sim 3 \mathrm{D})$ for a single water molecule is reached [13]. In order to verify this modification, we calculated the variation of the dipolar dielectric constant of bulk water with time using the modified TIP4P model; see Fig. 2(a). It is seen that the dielectric constant of bulk water lies in an acceptable range; i.e., it is between the dielectric constant of bulk water using the TIP4P and TIP4P/2005 models.
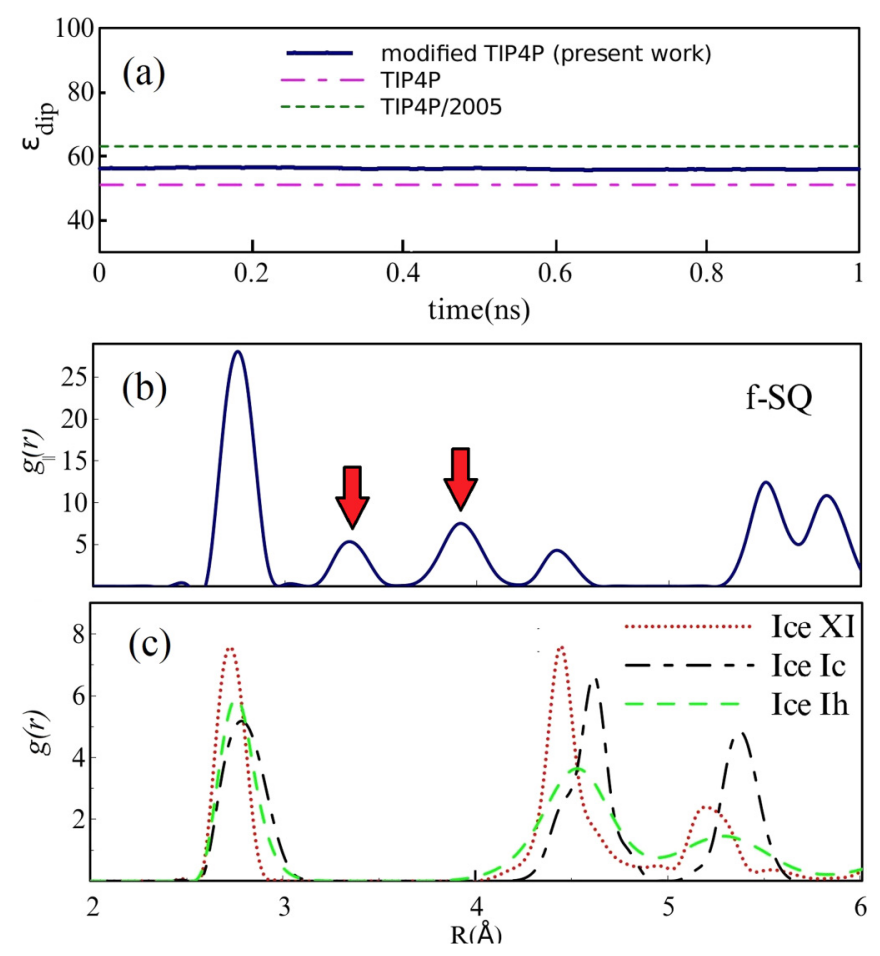

FIG. 2. (a) The variation of dielectric constant of bulk water with time using the modified TIP4P model in this study. (b) The in-plane radial distribution function of f-SQ 2D ice. Two arrows indicate the emergence of two peaks in f-SQ which are absent in bulk ices. (c) The radial distribution function of three typical bulk ices.

Periodic boundary conditions were applied along the $x, y$ directions and the confinement was along the $z$ direction. The particle-particle particle-mesh method was used to compute the long-range Coulomb interaction with a relative accuracy of $10^{-4}$. Water bond lengths and bond angles were fixed by the SHAKE algorithm [30]. In all MDSs a time step of 1 fs was chosen. Following the system relaxation (1 ns), the thermodynamical sampling was done up to $8 \mathrm{~ns}$ to ensure the smoothness of the converged dielectric constant. The temperature in MDSs for bulk ices (water) was set to be $80 \mathrm{~K}$ $(300 \mathrm{~K})$ because all of the studied bulk ices are stable at this temperature.

A microscopic picture of dielectric properties of $2 \mathrm{D}$ ices could be found by calculating the fluctuations of the total polarization of a system, $\vec{M}$, at finite temperature. By calculating different components of the total dipole moment, i.e., $M_{x}, M_{y}$, and $M_{z}$ after equilibration, one obtains different components of the molecular dielectric constant tensor as [31]

$$
\varepsilon_{\mathrm{dip}}^{\mu \nu}=\varepsilon_{\infty}+\frac{\sigma_{\mu \nu}^{2}}{\varepsilon_{0} k_{B} T V},
$$

where $\varepsilon_{\infty}$ is the optical dielectric constant and taken to be 1. Also, $\sigma_{\mu \nu}^{2}=\left\langle M_{\mu} M_{\nu}\right\rangle-\left\langle M_{\mu}\right\rangle\left\langle M_{\nu}\right\rangle$ while $\mu, v=x, y, z$ and $V$ is the volume of the system. Here, the time averaging was taken for more than $5 \mathrm{~ns}$ when the in-plane dielectric constant is converged. Note that Eq. (4) can only be used for homogeneous systems [15]. 


\section{Random-phase approximation: The frequency-dependent optical dielectric constant}

The optical dielectric function $\varepsilon_{\mathrm{el}}(\omega)$ was calculated using norm-conserving pseudopotentials, in the energy range of 0 to $30 \mathrm{eV}$. In order to increase accuracy for the dielectric function calculations, we used the $k$-point grid for $2 \mathrm{D}$ (bulk) ices as $12 \times 12 \times 1(12 \times 12 \times 12)$. The optical dielectric constant was calculated (the frequency-dependent part of the electronic dielectric constant or equivalently the electronic part of the dielectric function) within the framework of the random-phase approximation (RPA) based on DFT groundstate calculations. The mentioned dielectric function consists of frequency-dependent real $\left[\varepsilon_{\mathrm{el}}^{r}(\omega)\right]$ and imaginary parts $\left[\varepsilon_{\mathrm{el}}^{i}(\omega)\right]$. It is represented as

$$
\varepsilon_{\mathrm{el}}(\omega)=\varepsilon_{\mathrm{el}}^{r}(\omega)+i \varepsilon_{\mathrm{el}}^{i}(\omega) .
$$

The imaginary part of dielectric function $\left[\varepsilon^{i}(\omega)\right]$ can be calculated using the Kubo-Greenwood formalism [32]. Once we know the imaginary part, the real part can be obtained using the Kramers-Kronig relations (for more details see Ref. [33]). Note that the optical dielectric constant extracted from the RPA (at zero frequency) is compatible with that obtained from DFPT.

\section{RESULTS AND DISCUSSION}

\section{A. Electronic band structure}

The electronic band structure and partial density of states (PDOS) of 2D and bulk ices are shown in Fig. 3. We found the direct band gap of energy about 5.49, 5.55, and $5.12 \mathrm{eV}$ for f-SQ, b-SQ, and HEX 2D ices and 5.37 and $4.99 \mathrm{eV}$ for ice VIII and ice Ic, respectively. The electronic band structure for b-RH 2D ice, ice XI, and ice Ih confirms the indirect band gap. The corresponding energy gap for b-RH, ice XI, and ice Ih is 5.55, 5.06, and $5.08 \mathrm{eV}$, respectively. The PDOSs are shown on the right side of the panels of Fig. 3. It is seen that in contrast to the conduction bands, the $\mathrm{O}(\mathrm{H})$ atom contribution in the valance band is larger (negligible). Notice that the difference between the energy gap of 2D ices (and 2D $h$-BN) and bulk water (bulk $h$-BN) is small, i.e., $2 \%(4 \%)$, as compared to the difference between bulk TMDs and 2D TMDs; see Fig. 4. This is due to the weak hydrogen bond between water molecules in bulk and $2 \mathrm{D}$ ices as well as the insulating feature in all phases of water and $h$-BN. The TMDs have large reductions of energy gap while transiting from $3 \mathrm{D}$ to $2 \mathrm{D}$. The obtained energy gap, the electronic band structure, and the PDOS for ice VIII, ice XI, and ice Ih are in agreement with the results of Refs. [36-39].

Independently of water phase (including ice), the nearestneighbor distances between oxygen atoms of water molecules are almost the same, i.e., $2.8-3 \AA$; i.e., all the ice phases formed under very high pressures satisfy the so-called BernalFowler ice rules, where each water molecule has four hydrogen-bonded neighbors with a quasitetrahedral configuration with two short $\mathrm{O}-\mathrm{H}$ distances (the donated protons) and two long ones (the accepted protons). Transiting from bulk into $2 \mathrm{D}$ phase of ice, the crystalline structure with larger density can be formed where the nearest-neighbor distances are more or less the same but the preferential tetrahedral bonding geometry is different. In Figs. 2(b) and 2(c) we depict the in-plane radial distribution function for the O-O distance in f-SQ and the 3D-radial distribution function of three bulk ices (ice XI, ice Ic, and ice Ih). As can be seen, as expected the nearest-neighbor $\mathrm{O}-\mathrm{O}$ distances (first peaks) for all structures are equal. However, the second nearest neighbors [shown by arrows in Fig. 2(b)] are different. The latter causes a larger density of f-SQ [10] $\left(1.36 \mathrm{~g} \mathrm{~cm}^{-3}\right)$ as compared to bulk crystalline ices, i.e., $0.92 \mathrm{~g} \mathrm{~cm}^{-3}$ ice Ih and $0.92 \mathrm{~g} \mathrm{~cm}^{-3}$ ice XI.

On the other hand, the most well-known electrical conductivity mechanism for all phases of water/ice is the Grotthuss mechanism (GM), also known as proton jumping. In the GM an excess proton or proton defect diffuses through the hydrogen bond network of water molecules and a covalent bond between neighboring molecules is formed and broken continuously. Because of the same nearest-neighbor distance in bulk ice (bulk water) and those of 2D ice, the GM mechanism should be valid for 2D ices. Subsequently only an infinitesimal increase in the band gap 2D ices as compared to the bulk ices is seen. This was confirmed by our $a b$ initio simulations.

Moreover, $h$-BN is a wide band gap semiconductor with high thermal and chemical stability. In both bulk and monolayer $h$-BN, $\mathrm{N}$ atoms and $\mathrm{B}$ atoms are hybridized with $s p^{2}$ at the interlayer forming a honeycomb structure. In bulk $h$-BN there are weak interactions between each layer of $h$-BN, such as electrostatic interactions and van der Waals interactions, which cause the electrical band gap of both bulk $h-\mathrm{BN}$ and 2D $h$-BN to become more or less equal [40]. The transition from bulk into 2D TMDs also obeys the same general rule; i.e., the band gap of a 2D TMD is larger than that of bulk.

\section{B. Dielectric constant}

Here, we turn our attention to the main focus of this paper. The dielectric constants extracted from QE represent the combined dielectric constant of $2 \mathrm{D}$ ice and the surrounding large vacuum with a height $c$. In order to distill the dielectric constant of 2D ices, we eliminate the contribution of the vacuum using a capacitance model [18]. In fact, in the out-of-plane (in-plane) direction, the capacitance of the supercell extracted directly from the QE code $\left(\epsilon_{S C}\right)$ is the series (parallel) combination of vacuum capacitance and the 2D-ice capacitance. This helps us to find the out-of-plane and in-plane relative dielectric constant of 2D ices using below the equations [18]

$$
\varepsilon^{z z}=\left[1+\frac{c}{t}\left(\frac{1}{\varepsilon_{S C}}-1\right)\right]^{-1}, \varepsilon^{x x, y y}=1+\frac{c}{t}\left(\varepsilon_{S C}-1\right) .
$$

Here $t$ is the effective thickness of 2D ice.

In order to make sure that the used $c$ values are sufficiently large and the obtained $\varepsilon^{x x, y y, z z}$ were converged well, we performed several additional calculations and found corresponding $\varepsilon_{S C}$. The results show that when $c$ values change from 12 $\AA$ to $40 \AA$, the $\varepsilon^{x x, y y, z z}$ values only increase about $5 \%$. Thus, the used vacuum size (see Table I) is sufficiently large. 
(a)
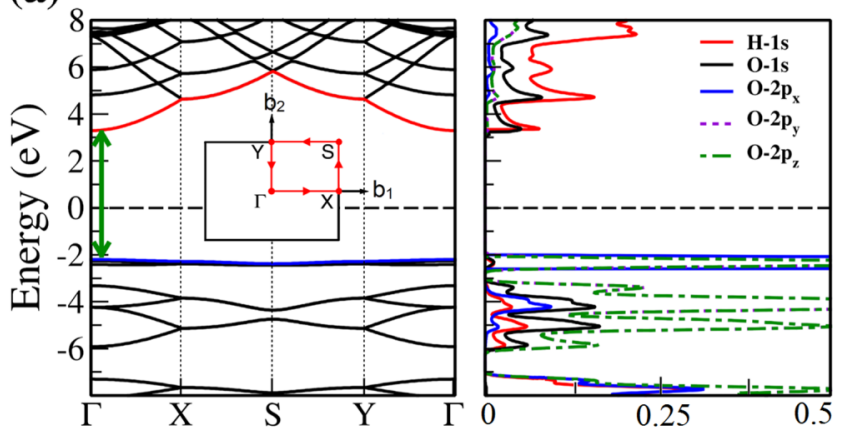

(c)
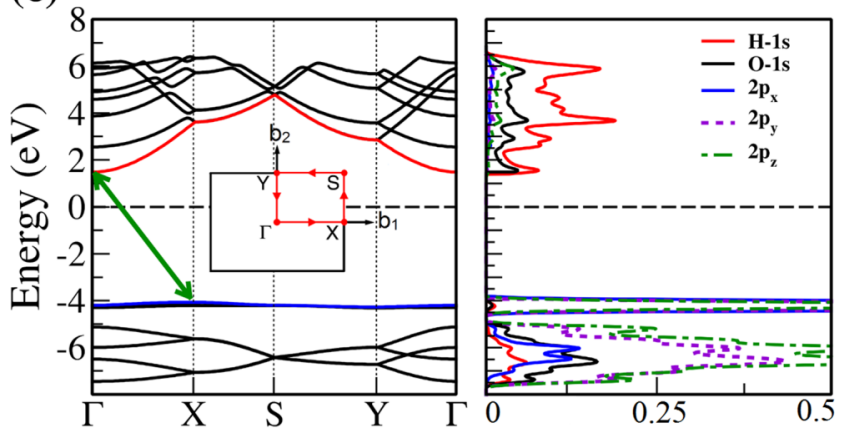

(e)

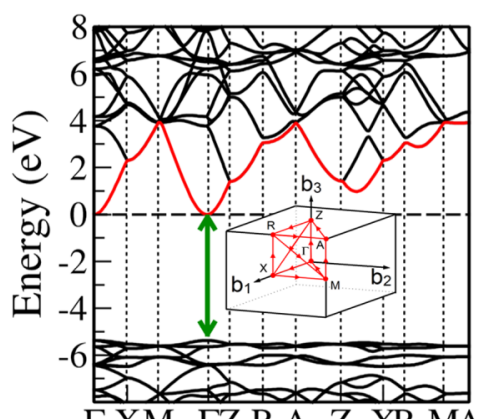

(g)

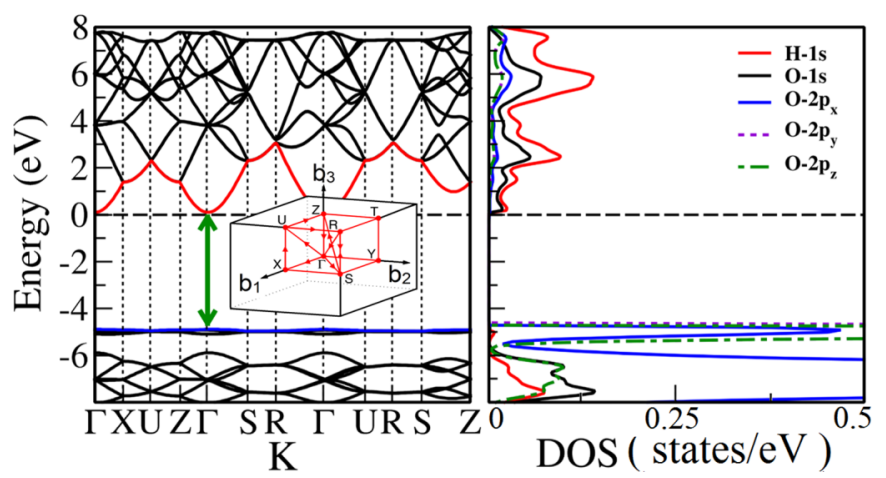

(b)
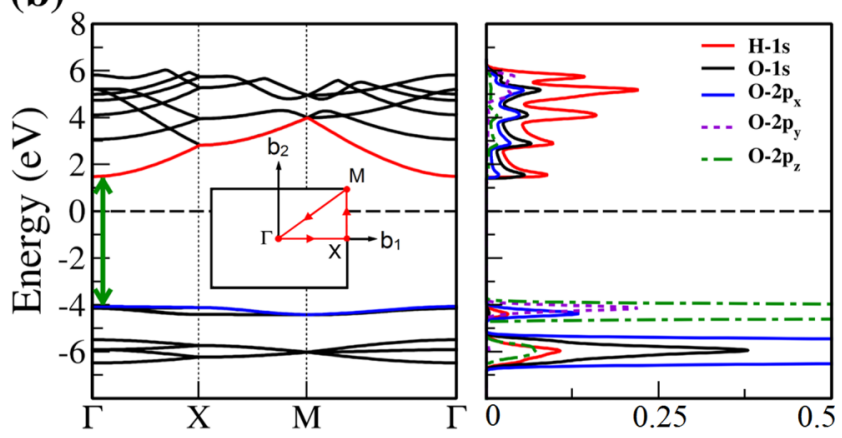

(d)
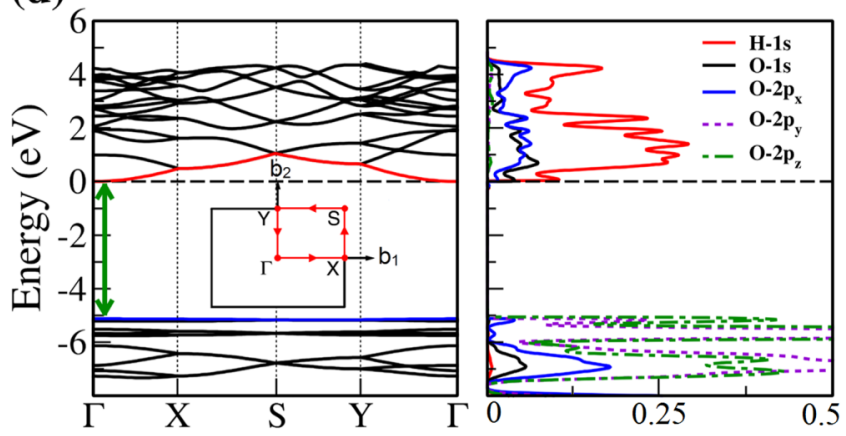

(f)
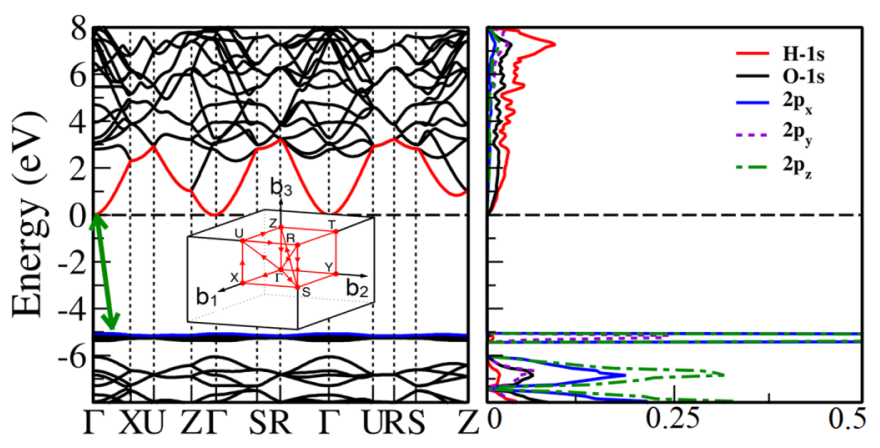

(h)

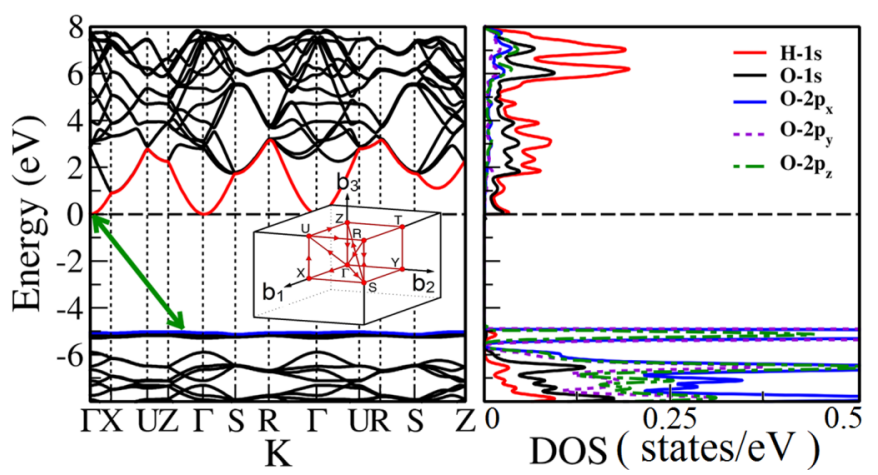

FIG. 3. The electronic band structure of (a) f-SQ, (b) b-SQ, (c) b-RH, (d) HEX, (e) ice VIII, (f) ice XI, (g) ice Ic, and (h) ice Ih and corresponding partial density of states. The arrows denote the type of band gap (direct or indirect) and the insets show the corresponding Brillouin zones.

Using the aforementioned correction, the results for the electronic $\left(\varepsilon_{\mathrm{el}}\right)$, ionic $\left(\varepsilon_{\mathrm{ion}}\right)$, and dipolar $\left(\varepsilon_{\mathrm{dip}}\right)$ dielectric con- stants for $2 \mathrm{D}$ ices are listed in Table I. The main findings are illustrated in Fig. 5 and are listed as follows: 


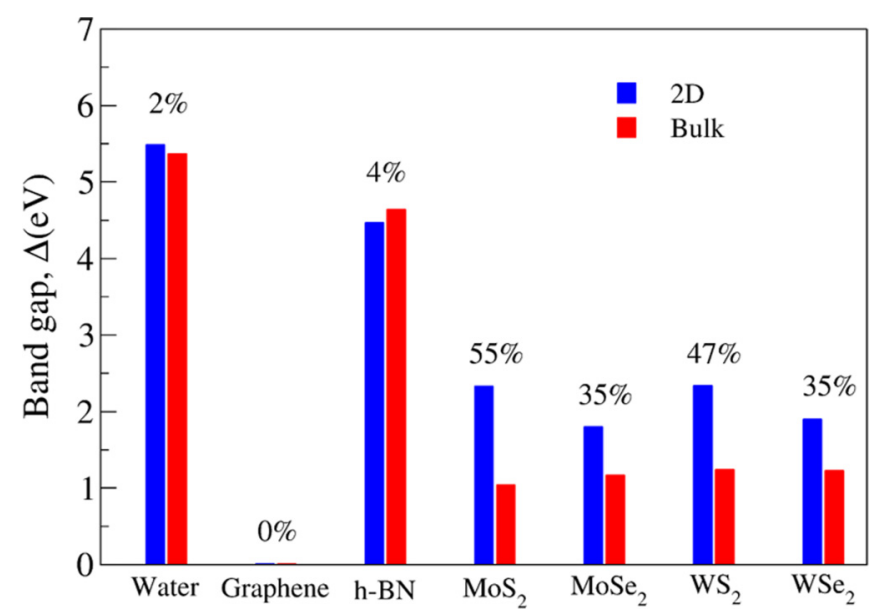

FIG. 4. The energy gap of bulk phase and 2D phase of various materials. The data for transition metal dichalcogenides were taken from Refs. [34,35].

(i) $\varepsilon_{\text {ion }}^{x x} \simeq \varepsilon_{\text {ion }}^{y y}$ for $2 \mathrm{D}$ ices except for b-RH ice in $\varepsilon_{\text {ion }}^{x x} \simeq$ $10 \varepsilon_{\text {ion }}^{y y}$, which is due to the remarkable in-plane anisotropy in the lattice structure of $\mathrm{b}-\mathrm{RH}$.

(ii) $\varepsilon_{\mathrm{el}}^{x x} \simeq \varepsilon_{\mathrm{el}}^{y y}$ for $2 \mathrm{D}$ (bulk) ices and is larger than (equal to) $\varepsilon_{\mathrm{el}}^{z z}$. The larger the $\varepsilon_{\mathrm{el}}^{x x}$ and $\varepsilon_{\mathrm{el}}^{y y}$, the greater the flatness of the structures. This is due to the confined electron clouds in quasi-2D space.

(iii) $\varepsilon_{\mathrm{el}}^{x x}$ and $\varepsilon_{\mathrm{el}}^{y y}$ of f-SQ ice are larger than those for other $2 \mathrm{D}$ ices. This might be due to the confining electron in the $2 \mathrm{D}$ plane and stronger response of system to an in-plane electric field, where for other 2D ices there is a small buckling in their geometry.

(iv) Because of the crystalline structure of all the studied crystalline 2D ices and bulk ices their dipolar contributions are remarkably smaller than that of bulk water.

(v) The electronic dielectric constants of 2D and bulk ices are smaller than for other 2D materials such as $\mathrm{MoS}_{2}, \mathrm{WSe}_{2}$, and $\mathrm{WS}_{2}$ [18]. The latter are attributed to the semiconducting nature of the TMDs rather than the insulating nature of $2 \mathrm{D}$ ices.

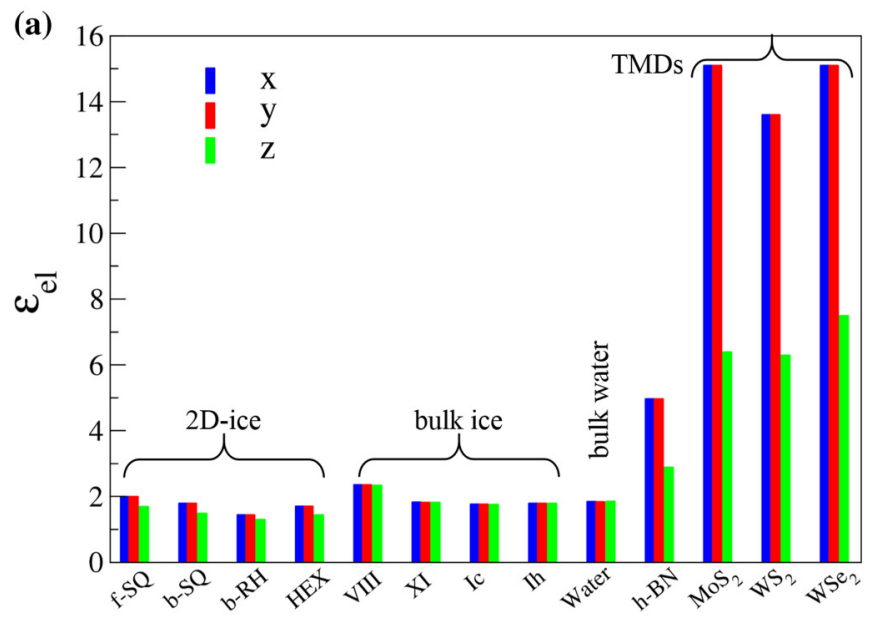

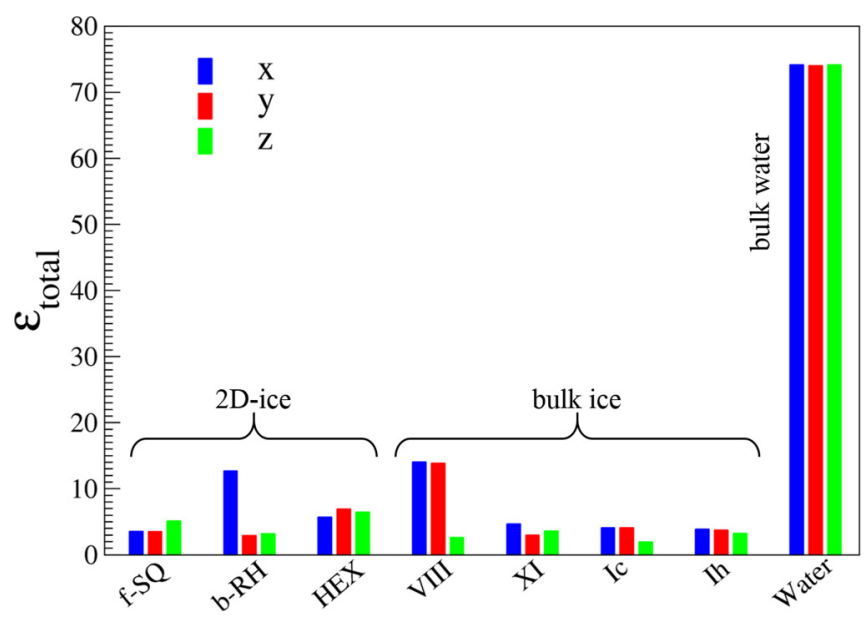

FIG. 6. The total dielectric constant $\left(\varepsilon_{\text {total }}\right)$ of 2D ices, bulk ices, and bulk water [41].

The aforementioned items are represented in Figs. 7(a) and 7(b). Consequently the total dielectric constants (Fig. 6) of the studied ices (except for b-RH and ice VIII) are smaller than 10 .

\section{Optical dielectric function}

In Fig. 7, the real $\left(\varepsilon^{r}\right)$ and imaginary $\left(\varepsilon^{i}\right)$ parts of the optical dielectric function are shown for $2 \mathrm{D}$ and bulk ices and bulk water. The results show the following:

(i) For $2 \mathrm{D}$ ices, $\varepsilon^{x x} \neq \varepsilon^{z z}$ in both real and imaginary parts, which is expected.

(ii) Because of the isotropic (anisotropic) lattice of the f-SQ and b-SQ ices (b-RH and HEX) for both real and imaginary parts, $\varepsilon_{\mathrm{el}}^{x x}(\omega)=\varepsilon_{\mathrm{el}}^{y y}(\omega)\left[\varepsilon_{\mathrm{el}}^{x x}(\omega) \neq \varepsilon_{\mathrm{el}}^{y y}(\omega)\right]$.

(iii) Despite the bulk cubic ice structures (ice VIII and ice Ic) and the bulk hexagonal ice structures (ice $\mathrm{XI}$ and ice Ih), the dielectric functions are almost equal, i.e., $\varepsilon_{\mathrm{el}}^{x x}(\omega) \simeq \varepsilon_{\mathrm{el}}^{y y}(\omega) \simeq \varepsilon_{\mathrm{el}}^{z z}(\omega)$, for both real and imaginary parts.

The absorption behavior of ices can be understood by analyzing the imaginary part of the dielectric function. Also,

(b)

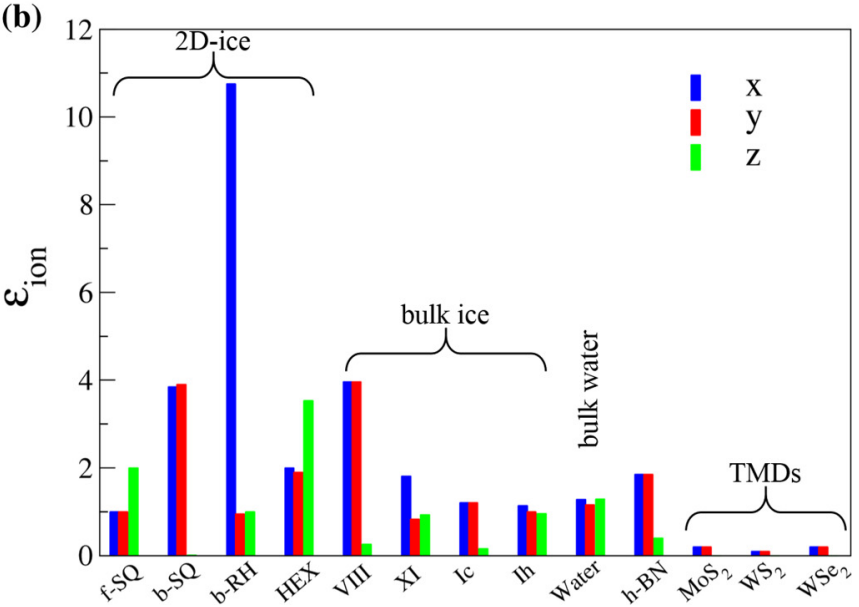

FIG. 5. The electronic (a) and ionic (b) dielectric constant of 2D ices, bulk ices, and TMDs. The data for TMDs were taken from Ref. [18]. 

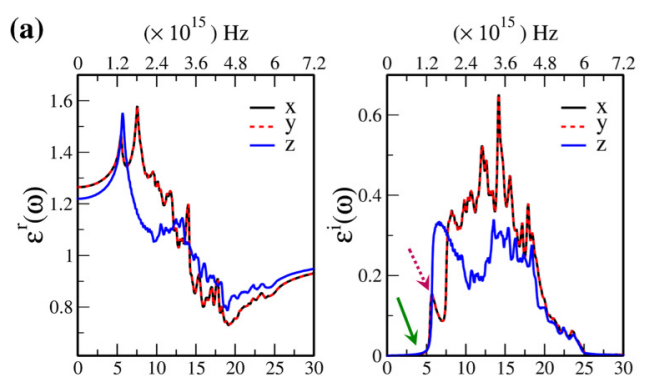

(c)
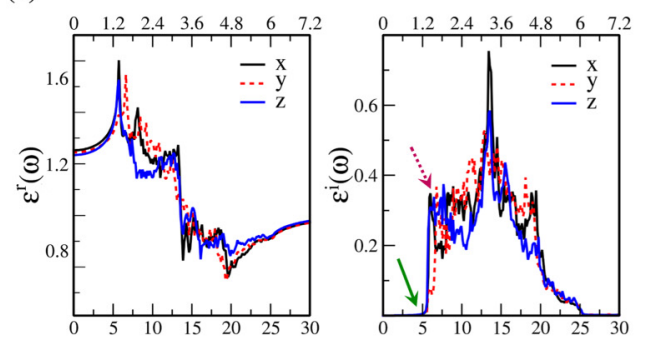

(e)
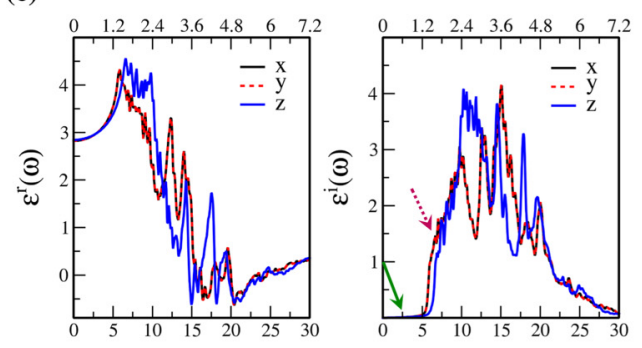

(g)
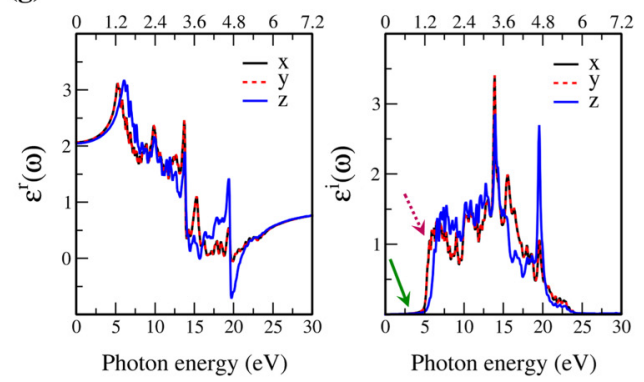

(b)

$\left(\times 10^{15}\right) \mathrm{Hz}$
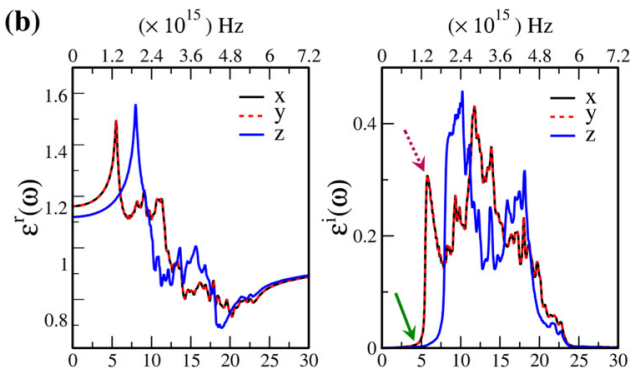

(d)
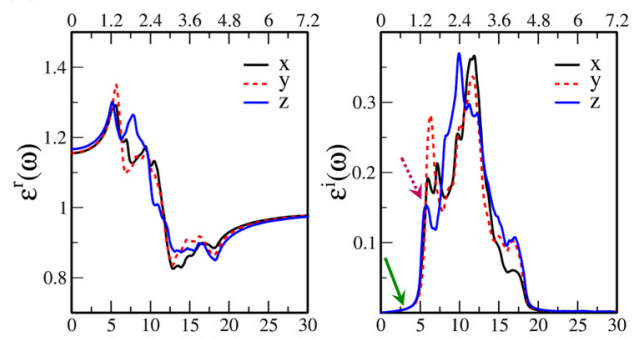

(f)
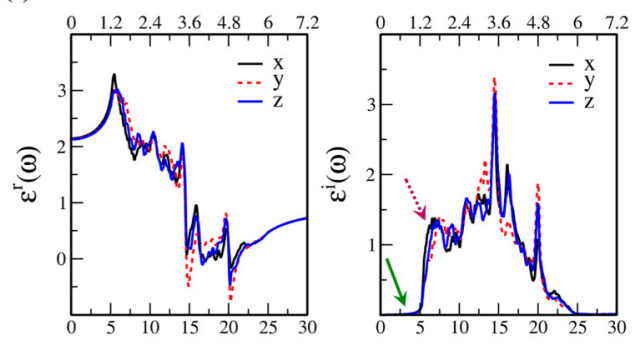

(h)

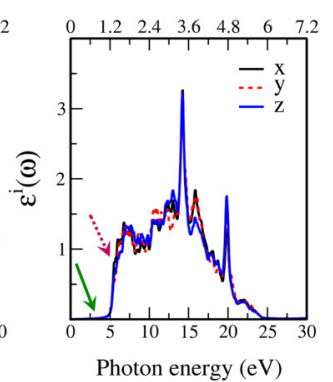

(i)

$\left(\times 10^{15}\right) \mathrm{Hz}$
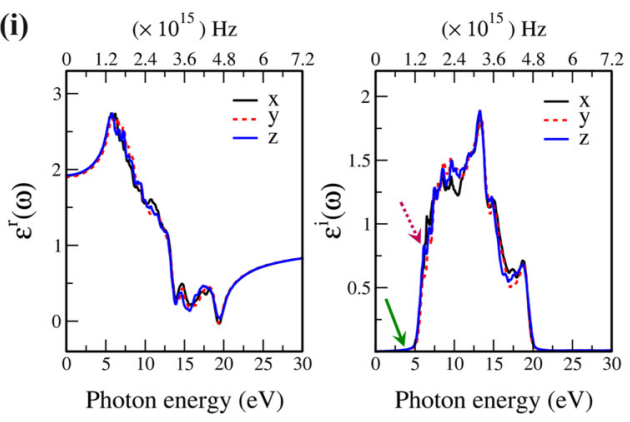

FIG. 7. The real and imaginary part of dielectric function for (a) f-SQ, (b) b-SQ, (c) b-RH, (d) HEX, (e) ice VIII, (f) ice XI, (g) ice Ic, (h) ice Ih, (i) bulk water. The green-solid (pink-dashed) arrows refer to the optical gap (energy gap).

the absorption edge gives the optical gap $\left(\Delta_{o}\right)$. Moreover, the peaks in the $\varepsilon^{i}(\omega)$ function are related to interband transitions in the electronic band structure. For instance, the first peak corresponds to the energy gap $(\Delta)$. Indeed, the first critical point calculated in $\varepsilon_{\mathrm{el}}^{i}(\omega)$ is related to the transition from valence band maximum to the conduction band minimum, i.e., the energy band gap. 

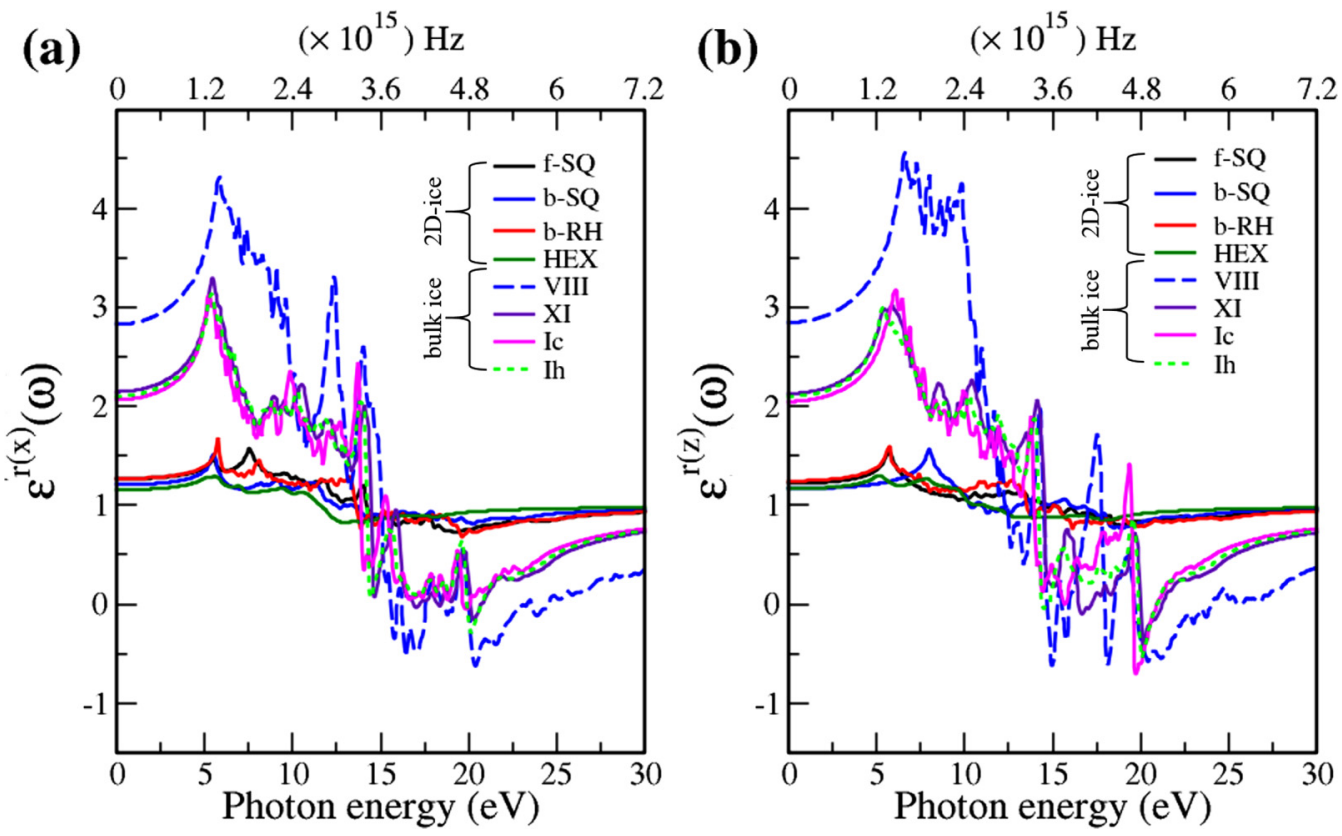

FIG. 8. The real dielectric function of 2D and bulk ices: (a) in-plane ( $x x, y y$ components) and (b) out-of-plane ( $z z$ component).

The green-solid and pink-dashed vertical arrows in Fig. 7 denote the optical gap and energy gap. In general, the optical gap is equal to the electronic band gap minus the exciton binding energy. In other words, the optical gap and electronic gap should be equal if the many-body perturbation theory is not taken into account. Therefore, our obtained difference between optical gap and electronic band gap is due to the smearing applied in the implemented Kubo-Greenwood approach [42]. Moreover, note that $\varepsilon^{r}(\omega=0)$ in Fig. 7 and $\varepsilon_{\text {el }}^{0}$ listed in Table I apparently are different. However, the difference originates in the two different methods that we employed to calculate them. The first one is the microscopic dielectric function that was obtained by using the RPA and the second one is the macroscopic dielectric function that was obtained by using DFPT. Thus, they coincide if the same method is used to obtain them.

To compare the optical properties of $2 \mathrm{D}$ and bulk ices, we demonstrate in Fig. 8 (Fig. 9) the real (imaginary) dielectric functions. The results show the following:

(i) The real part of the dielectric function has larger values in bulk ices as compared to 2D ices in the low-energy region. This is due to the fact that electrons are distributed in 3D space in bulk ice. Moreover, the peaks are larger in 2D ices as compared to the bulk ices.

(ii) The negative values of the real dielectric function of bulk ices correspond to the largest electromagnetic wave reflection. In fact the inductive properties will dominate in this range of energies with the negative values of the real dielectric function. In simple words, at these energy ranges (e.g., in ice VIII the energy range $15-20 \mathrm{eV}$ has the negative dielectric function) the electric displacement vector and the electric field vector have opposite directions.

(iii) There is a redshift (a move toward the lower-energy region) of the major peaks of $\varepsilon_{\mathrm{el}}^{i}(\omega)$ for bulk ices in compar- ison to $2 \mathrm{D}$ ices. In all the studied ices, the prominent peaks in $\varepsilon_{\mathrm{el}}^{i}(\omega)$ correspond to optical transmission which is mainly due to the interband transition from the $p$ valence bands to the $s$ conduction bands [i.e., the O- $p$ orbital below the Fermi level (valence band) and H-s orbital above the Fermi level (conduction band)]. The PDOS of each system is shown in the right-hand-side panels of Fig. 3.

(iv) The absorption energy ranges for $2 \mathrm{D}$ and bulk ices are in the ultraviolet spectra $(>3.2 \mathrm{eV})$ and visible spectra (between 2 and $3.2 \mathrm{eV}$ ), respectively.

The obtained dielectric functions for ice VIII, ice XI, ice Ic, ice Ih, and bulk water are consistent with the results of Refs. [36,43], Ref. [39], and Refs. [44,45], respectively. Note that $\varepsilon^{r}(\omega=0)$ in Fig. 7 and $\varepsilon_{\text {el }}^{0}$ listed in Table I apparently are different. However, the difference originates in the two different methods that we employed to calculate each of them. The first one corresponds to the microscopic dielectric function which was obtained by using the RPA and the second one corresponds to the macroscopic dielectric function which was obtained by using DFPT.

\section{Mechanical stiffness of 2D ice}

It is insightful to calculate mechanical stiffness of a typical 2D ice (the results are selectively presented for f-SQ). We performed an additional calculation for determining the Young's modulus and Poisson's ratio of f-SQ ice. We applied both uniaxial and biaxial strains and found the corresponding energies $E_{u}$ and $E_{b}$, for uniaxial and biaxial strained systems. Next, the best fits on the two equations $E_{b}=2 b u_{b}^{2}$ and $E_{u}=$ $\frac{1}{2}(b+\mu) u_{u}^{2}$ helped us to obtain the 2D-bulk modulus $b$ and 2D-shear modulus $\mu$. Here $u_{u}$ and $u_{b}$ are the applied uniaxial and biaxial strains, respectively. Consequently, the Young's modulus $\left(Y_{2 d}\right)$ and Poisson's ratio $\left(\nu_{2 d}\right)$ can be computed 
(a)

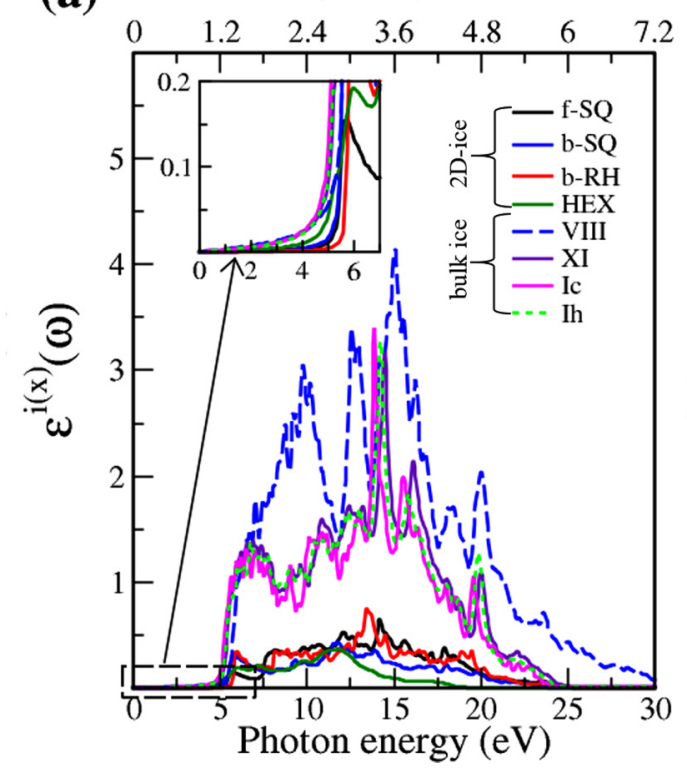

(b)

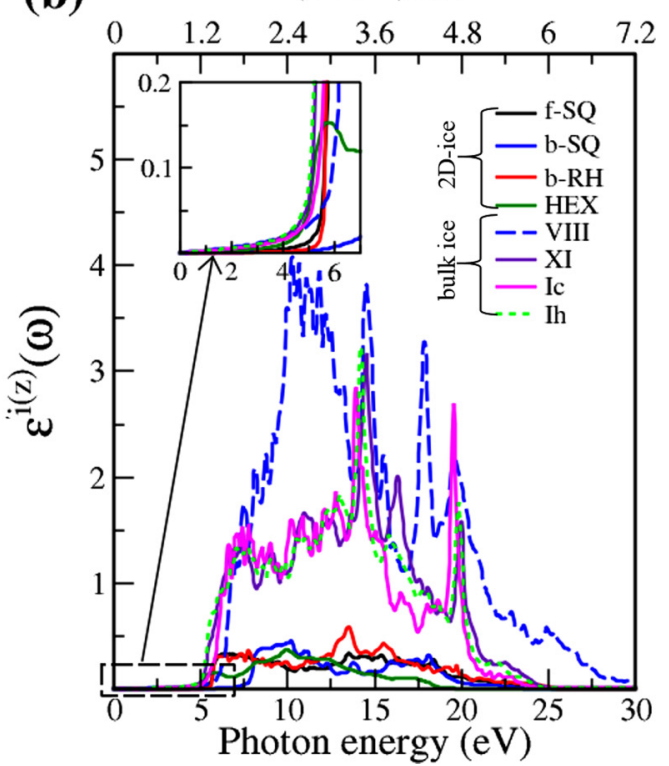

FIG. 9. The imaginary dielectric function of 2D and bulk ices: (a) in-plane ( $x x, y y$ components) and (b) out-of-plane ( $z z$ component).

[46]:

$$
Y_{2 d}=\frac{4 b \mu}{b+\mu}, \quad \nu_{2 d}=\frac{b-\mu}{b+\mu} .
$$

The results are $Y_{\mathrm{f}-\mathrm{SQ}}=3.6 \mathrm{~N} / \mathrm{m}$ and $v=0.6$. The Young's modulus of $2 \mathrm{D}$ ice is 2 orders of magnitude smaller than the one for graphene, i.e., $340 \mathrm{~N} / \mathrm{m}$ [47]. This is due to the weak hydrogen bonds in $2 \mathrm{D}$ ices compared to the strong planar covalent bonds in graphene. The corresponding Poisson's ratio of $2 \mathrm{D}$ ice is larger than that for graphene $(0.3)$. The obtained Young's modulus (Poisson's ratio) for 2D ice is more or less equal to the bulk modulus of bulk ice. In Table II, we list the Young's modulus/bulk modulus and Poisson's ratio of f-SQ/bulk crystalline ice. To convert the $\mathrm{N} / \mathrm{m}$ units in $Y_{2 d}$ to $\mathrm{Pa}$ units, one may use the simple relation $Y_{3 d}=Y_{2 d} / t_{0}$, where $t_{0}=3 \AA$ is the effective thickness of f-SQ ice (see Table I).

\section{CONCLUSIONS}

In summary, we found that the energy gap in $\mathrm{f}-\mathrm{SQ}, \mathrm{b}-\mathrm{SQ}$, and HEX 2D-ice structures and cubic bulk ices (ice VIII and ice Ic) is direct, whereas b-RH 2D ice and hexagonal bulk ices (ice XI and ice Ih) have an indirect band gap. The underlying

TABLE II. The Young's modulus and Poisson's ratio of f-SQ and bulk modulus $(B)$ of three bulk crystalline ices.

\begin{tabular}{lcc}
\hline \hline Ice & $Y(\mathrm{GPa})$ & $v$ \\
\hline f-SQ & 12 & 0.6 [present work] \\
& & \\
Ice & $B(\mathrm{GPa})$ & $v$ \\
Ice VIII [48] & 18 & $0.28[49]$ \\
Ice VII [48] & 13 & $0.325[49]$ \\
Ice Ih [48] & 8.5 & \\
\hline \hline
\end{tabular}

lattice structure and symmetry significantly influence the ionic and dipolar terms, but their effects on the electronic dielectric constant are negligible.

We found the total out-of-plane dielectric constant is larger than 2 for all the studied 2D ices (except b-SQ) and bulk ices, i.e., $\varepsilon_{\text {total }}^{z z}>2.0$ (see Table I). This clearly shows that the lattice structure of the confined water in the recent experiment [12] is not any of the lattice structures of the studied 2D ices here, and likely has random structure. On the other hand the small out-of-plane dielectric constant of about $\simeq 2.1$ (for nanoconfined water in channels with heights $h \sim 10 \AA$ ) [12] should not correspond to a monolayer water. Beyond $\sim 15 \AA$ a nonlinear increase (up to the bulk value) in the dielectric constant was found [12]. Therefore, we do not expect to recover experimental data when studying monolayer crystalline ice. It is also interesting to know that there are no reliable experimental data for the in-plane dielectric constant of confined water at subnanometer scale slits. Equivalently, the density of trapped water may be much lower than the bulk density [12]. This motivated us to determine the dielectric properties of amorphous 2D ice in an ongoing study.

The optical gap of 2D ices is found to be larger than that of bulk ices. The absorption energy ranges for $2 \mathrm{D}$ and bulk ices are in the ultraviolet spectra $(>3.2 \mathrm{eV})$ and visible spectra (between 2 and $3.2 \mathrm{eV}$ ), respectively. Generally, in bulk materials the presence of a large number of atoms and the merging of many adjacent energy levels result in the well-known energy conduction and valance bands. In 2D materials, due to the smaller number of atoms, the number of energy levels decreases, giving the narrower energy bands. As a result, the energy band gap will increase (the difference between valance band and conduction band). Also, the larger band gap in 2D ice will cause a shift of absorption spectrum toward lower wavelength (larger energies). In other words, there is redshift in the peaks of $\varepsilon^{i}$ of bulk ices in comparison to those of 2D ices [50]. 
We believe that our findings not only provide a theoretical background for understanding the different aspects of dielectric properties of confined water, but also give insights into the light absorption mechanism and corresponding absorption energy range of confined water which might be necessary for further experimental characterizations of $2 \mathrm{D}$ ices.

\section{ACKNOWLEDGMENTS}

We acknowledge Nassim Kangarlou for critically reading our paper and giving us useful comments. We would like to acknowledge high-performance computing support from Shahid Rajaee TT University sponsored by Iran National Science Foundation (INSF).
[1] G. Algara-Siller, O. Lehtinen, F. C. Wang, R. R. Nair, U. Kaiser, H. A. Wu, A. K. Geim, and I. V. Grigorieva, Nature (London) 519, 443 (2015).

[2] J. Chen, G. Schusteritsch, C. J. Pickard, C. G. Salzmann, and A. Michaelides, Phys. Rev. Lett. 116, 025501 (2016).

[3] J. Chen, A. Zen, J. G. Brandenburg, D. Alfè, and A. Michaelides, Phys. Rev. B 94, 220102(R) (2016).

[4] M. Neek-Amal, F. M. Peeters, Irina V. Grigorieva, and A. K. Geim, ACS Nano 10, 3685 (2016).

[5] M. S. Fernandez, M. Neek-Amal, and F. M. Peeters, Phys. Rev. B 92, 245428 (2015).

[6] V. Satarifard, M. Mousaei, F. Hadadi, J. Dix, M. S. Fernandez, P. Carbone, J. Beheshtian, F. M. Peeters, and M. Neek-Amal, Phys. Rev. B 95, 064105 (2017).

[7] M. Neek-Amal et al., Appl. Phys. Lett. 113, 083101 (2018).

[8] H. Ghorbanfekr-Kalashami, K. S. Vasu, R. R. Nair, F. M. Peeters, and M. Neek-Amal, Nat. commun. 8, 1 (2017).

[9] W. Zhou, K. Yin, C. Wang, Y. Zhang, T. Xu, A. Borisevich, L. Sun, J. C. Idrobo, M. F. Chisholm, S. T. Pantelides, R. F. Klie, and A. R. Lupini, Nature (London) 528, E1 (2015).

[10] M. Sobrino Fernandez, F. M. Peeters, and M. Neek-Amal, Phys. Rev. B 94, 045436 (2016).

[11] R. Zangi and A. E. Mark, Phys. Rev. Lett. 91, 025502 (2003).

[12] L. Fumagalli, A. Esfandiar, R. Fabregas, S. Hu, P. Ares, A. Janardanan, Q. Yang, B. Radha, T. Taniguchi, K. Watanabe, G. Gomila, K. S. Novoselov, and A. K. Geim, Science 360, 1339 (2018)

[13] J. L. Aragones, L. G. MacDowell, and C. Vega, J. Phys. Chem. A 115, 5745 (2011).

[14] Daniel C. Elton, Ph.D. thesis, Stony Brook University, 2016.

[15] A. Schlaich, Ernst W. Knapp, and Roland R. Netz, Phys. Rev. Lett. 117, 048001 (2016).

[16] V. Ballenegger and J.-P. Hansen, J. Chem. Phys. 122, 114711 (2005).

[17] H. Itoh and H. Sakuma, J. Chem. Phys. 142, 184703 (2015).

[18] A. Laturia, M. L. Van de Put, and W. G. Vandenberghe, npj 2D Mater. Appl. 2, 6 (2018).

[19] See http://www1.lsbu.ac.uk/water/martin_chaplin.html.

[20] N. E. Brener, Phys. Rev. B 12, 1487 (1975).

[21] P. Giannozzi, S. Baroni, N. Bonini, M. Calandra, R. Car, C. Cavazzoni, D. Ceresoli, G. L. Chiarotti, M. Cococcioni, I. Dabo, A. D. Corso, S. deGironcoli, S. Fabris, G. Fratesi, R. Gebauer, U. Gerstmann, C. Gougoussis, A. Kokalj, M. Lazzeri, L. Martin-Samos, N. Marzari, F. Mauri, R. Mazzarello, S. Paolini, A. Pasquarello, L. Paulatto, C. Sbraccia, S. Scandolo, G. Sclauzero, A. P. Seitsonen, A. Smogunov, P. Umari, and R. M. Wentzcovitch, J. Phys.: Condens. Matter 21, 395502 (2009).
[22] M. Dion, H. Rydberg, E. Schroder, D. C. Langreth, and B. I. Lundqvist, Phys. Rev. Lett. 92, 246401 (2004).

[23] C. A. Rozzi, D. Varsano, A. Marini, E. K. U. Gross, and A. Rubio, Phys. Rev. B 73, 205119 (2006).

[24] T. Sohier, M. Calandra, and F. Mauri, Phys. Rev. B 96, 075448 (2017).

[25] S. Baroni, S. de Gironcoli, A. D. Corso, and P. Gionazzi, Rev. Mod. Phys. 73, 515 (2001).

[26] X. Gonze and C. Lee, Phys. Rev. B 55, 10355 (1997).

[27] S. Plimpton, J. Comput. Phys. 117, 1 (1995).

[28] We found that b-SQ is unstable in our classical molecular dynamics simulation.

[29] J. L. F. Abascal and C. Vega, J. Chem. Phys. 123, 234505 (2005).

[30] J. P. Ryckaert, G. Ciccotti, and H. J. Berendsen, J. Comput. Phys. 23, 327 (1977).

[31] C. Zhang, F. Gygi, and G. Galli, J. Phys. Chem. Lett. 4, 2477 (2013).

[32] G. J. Morgan and H. B. Ghassib, Solid State Commun. 67, 1035 (1988).

[33] D. M. Roessler, Br. J. Appl. Phys. 16, 1119 (1965).

[34] J. Gusakova, X. Wang, L. L. Shiau, A. Krivosheeva, V. Shaposhnikov, V. Borisenko, V. Gusakov, and B. K. Tay, Phys. Status Solidi B 214, 700218 (2017).

[35] M. Topsakal, E. Aktürk, and S. Ciraci, Phys. Rev. B 79, 115442 (2009).

[36] R. Xu, Z. Liu, Y. Ma, T. Cui, B. Liu, and G. Zou, arXiv:0801.0400.

[37] A. Hermann and P. Schwerdtfeger, Phys. Rev. Lett. 106, 187403 (2011).

[38] C. Fang, W. F. Li, R. S. Koster, and J. Klime, Phys. Chem. Chem. Phys. 17, 365 (2015).

[39] M. de Koning, A. Fazzio, A. J. R. da Silva, and A. Antonelli, Phys. Chem. Chem. Phys. 18, 4652 (2016).

[40] Jingang Wang, Fengcai Ma, Wenjie Liang, and Mengtao Sun, Mater. Today Phys. 2, 6 (2017).

[41] Notice that we subtract $\varepsilon_{\infty}$ from $\varepsilon_{\text {total }}$ in order to avoid double counting, since $\varepsilon_{\infty}$ appears in both $\varepsilon_{\text {dip }}$ and $\varepsilon_{\mathrm{el}}$.

[42] Benoît Sklénard, Alberto Dragoni, François Triozon, and Valerio Olevano, Appl. Phys. Lett. 113, 172903 (2018).

[43] R. Xu, Z. Liu, Y. Ma, T. Cui, B. Liu, and G. Zou, arXiv:0801.0187.

[44] V. Garbuio, M. Cascella, L. Reining, R. Del Sole, and O. Pulci, Phys. Rev. Lett. 97, 137402 (2006).

[45] V. Garbuio, M. Cascella, I. Kupchak, O. Pulci, and A. P. Seitsonen, J. Chem. Phys. 143, 084507 (2015).

[46] K. V. Zakharchenko, M. I. Katsnelson, and A. Fasolino, Phys. Rev. Lett. 102, 046808 (2009). 
[47] Changgu Lee, Xiaoding Wei, Jeffrey W. Kysar, and James Hon, Science 321, 385 (2008).

[48] S. Klotz, K. Komatsu, H. Kagi, K. Kunc, A. Sano-Furukawa, S. Machida, and T. Hattori, Phys. Rev. B 95, 174111 (2017).
[49] G. H. Shaw, J Chem. Phys. 84, 5862 (1986).

[50] Yiling Yu, Yifei Yu, Yongqing Cai, Wei Li, Alper Gurarslan, Hartwin Peelaers, David E. Aspnes, Chris G. Van de Walle, Nahan V. Nguyen, and Yong-Wei Zhang, Sci. Rep. 5, 16996 (2015). 